\title{
Association of Growth and Differentiation Factor 15 in Rheumatoid Arthritis
}

\author{
Yan-Wei He', Cheng-Song $\mathrm{He}^{2}$ \\ 'Department of Orthopaedics, Affiliated Hospital of Southwest Medical University, Luzhou, Sichuan, 646000, People's Republic of China; ${ }^{2}$ Department \\ of Rheumatology and Immunology, Affiliated Hospital of Southwest Medical University, Luzhou, Sichuan, 646000, People's Republic of China \\ Correspondence: Cheng-Song He, Department of Rheumatology and Immunology, Affiliated Hospital of Southwest Medical University, Luzhou, \\ Sichuan, 646000, People's Republic of China, Email I3700980878@I63.com
}

Purpose: Rheumatoid arthritis (RA) is an inflammatory rheumatic disease, which has been demonstrated to correlate with mutated genetics. Growth and differentiation factor 15 (GDF-15) is a member of the transforming growth factor- $\beta$ superfamily and is expressed in different organs, tissues and immune cells. To date, limited studies have evaluated plasma levels of GDF-15 in RA patients, and whether GDF-15 gene polymorphisms correlate with RA risk in the Chinese Han population has not been clarified.

Patients and Methods: This case-control study recruited 910 age- and sex-matched RA patients and healthy controls. Plasma levels of GDF-15 were examined by enzyme linked immunosorbent assay, and polymorphisms (rs1055150, rs1058587, rs3787023, and rs4808793) were genotyped by KASP method.

Results: RA patients had higher levels of GDF-15 as compared to that in healthy controls. Patients with positive CRP also showed higher levels of GDF-15 when compared to that in patients with negative CRP. Levels of GDF-15 correlated with disease activity score. Frequencies of GG, GC, GG+GC genotypes and G allele in GDF-15 gene rs 1058587 were significantly elevated in RA patients compared to controls. Frequencies of CC genotype and C allele in GDF-15 gene rs3787023 were higher in RA patients compared to controls. Other polymorphisms did not correlate with RA susceptibility. Moreover, the four polymorphisms were not correlated with levels of GDF-15.

Conclusion: Plasma levels of GDF-15 were elevated in RA patients and GDF-15 gene polymorphisms were related to RA risk in the Chinese Han population.

Keywords: rheumatoid arthritis, growth and differentiation factor 15, polymorphism, pathogenesis

\section{Introduction}

Rheumatoid arthritis (RA) is a chronic inflammatory autoimmune disease. ${ }^{1}$ This disease is characterized by large autoantibodies production, paired with tender and swollen joints. To date, the clear pathogenesis of RA has not been clarified. However, inflammatory cytokines genetics have been widely discussed and are accepted to play significant roles in the development of this complex disorder. ${ }^{2}$

Growth and differentiation factor 15 (GDF-15) belongs to the transforming growth factor- $\beta$ (TGF- $\beta$ ) superfamily and was first identified in $1997 .^{3}$ This superfamily has more than 30 members. GDF-15 circulates as a $\sim 25 \mathrm{kDa}$ homodimer comprising two 112 amino acid polypeptide chains. ${ }^{4}$ Human GDF-15 gene locates at chromosome 19p12.1-13.1. GDF15 expresses at different organs or tissues, such as the placenta, kidney, or lung, and different immune cells express GDF15 , such as the macrophage or T-cell. ${ }^{5}$ Several studies have discussed GDF-15 gene polymorphisms with human diseases. ${ }^{6-8}$ For example, carriers of the GC genotype in rs 1058587 polymorphism had a lower risk of sporadic prostate cancer and of familial prostate cancer than the CC genotype carriers. ${ }^{8}$ There was a strong association between serum levels of GDF-15 and neoplastic progression, and determination of GDF-15 gene rs 1058587 genotypes may have clinical usage in management of colorectal carcinoma patients. ${ }^{7}$ Recently, studies have shown that serum levels of GDF-15 were higher in RA patients, correlating with disease activity. However, whether plasma levels of GDF-15 were abnormal in 
RA patients needs to be clarified. In addition, whether GDF-15 gene polymorphisms were related to RA risk in the Chinese Han population needs to be discussed.

\section{Methods}

\section{Patients}

This study recruited 455 RA patients and 455 healthy controls from the department of Rheumatology and Immunology, department of Orthopaedics, affiliated hospital of Southwest Medical University. RA patients were diagnosed according to the American College of Rheumatology and European League Against Rheumatism (ACR/EULAR) classification criteria. ${ }^{9}$ RA patients were either newly diagnosed without any treatment or had been diagnosed before but had not used any medication in the last 3 months. Healthy controls were recruited from the Health Management Center in the affiliated hospital of Southwest Medical University. RA patients are of Chinese Han origin. Among the patients, 112 were male and 343 were female, while there were 116 male and 339 female healthy controls. Patients and controls were agematched as well (for patients, 56.00 (49.00-64.00) years; for controls, 55.00 (48.00-65.00) years). This study was approved by Ethnic Research Commit of affiliated hospital of Southwest Medical University and followed the 1964 Helsinki declaration and its later amendments. Informed consent was collected from all the patients and healthy controls.

Evaluation of disease activity for RA patients was confirmed by disease activity score 28 (erythrocyte sedimentation rate) (DAS28 (ESR).$^{10}$ For RA patients, disease duration, number of tender joints, number of swollen joints, levels of C-reactive protein (CRP), ESR, rheumatoid factor (RF), antinuclear antibody (ANA), and score of health assessment questionnaire (HAQ) were obtained from all the RA patients (Table 1). However, some of the RA patients had no information about IgG, IgA, IgM, or anti-cyclic citrullinated peptide antibodies (anti-CCP) (data not shown). In RA patients, a disease duration of more than 18 months was considered as long disease and a disease duration of less than 18 months was recognized as early disease. ${ }^{11}$ ESR was evaluated according to the distance from which the red blood cells sink at the end of the first hour (h). Plasma levels of CRP, RF were evaluated by scatter turbidimetry (Siemens Healthcare Diagnostics Products, GmbH, Germany). Levels of CRP larger than $8 \mathrm{mg} / \mathrm{L}$ were recognized as a positive result. ANA was detected by line blot techniques (Euroimmun, Lubeck, Germany).

\section{Detection of Plasma Levels of GDF-I5}

About $5 \mathrm{~mL}$ of peripheral blood was obtained from each patient and healthy control. After centrifugation, plasma and peripheral blood mononuclear cells (PBMC) were collected separately, and stored at $-80^{\circ} \mathrm{C}$ until usage. When examining plasma levels of GDF-15, the enzyme linked immunosorbent assay (ELISA) kits were purchased from CUSABIO, Newark, DE. The protocol to examine the levels of GDF-15 was described in brief. First, $100 \mu \mathrm{L}$ working standards or samples were approximately added into the 96-well assay plate, incubating for $2 \mathrm{~h}$ at $37^{\circ} \mathrm{C}$. Second, removing the liquid,

Table I Characteristics of Patients with Rheumatoid Arthritis and Healthy Controls

\begin{tabular}{|l|c|c|c|}
\hline Characteristics & RA Patients & Healthy Controls & P-value \\
\hline Age (years) & $56.00(49.00-64.00)$ & $55.00(48.00-65.00)$ & 0.349 \\
Sex (male/female) & $112 / 343$ & $116 / 339$ & 0.760 \\
Disease duration (years) & $0.50(0.00-6.50)$ & - & - \\
Tender joints (n) & $10.50(4.00-18.00)$ & - & - \\
Swollen joints (n) & $6.00(2.00-12.00)$ & - & - \\
CRP (mg/L) & $20.6(5.20-49.55)$ & - & - \\
RF (IU/mL) & $117.65(35.70-343.85)$ & - & - \\
ESR (mm/H) & $60.50(31.75-96.00)$ & - & - \\
ANA (+) (n, \%) & $225(49.43)$ & - & - \\
DAS28 (ESR) & $6.12(5.26-7.16)$ & - & - \\
HAQ & $17.00(8.00-12.00)$ & - & - \\
\hline
\end{tabular}

Abbreviations: RA, rheumatoid arthritis; CRP, C-reactive protein; RF, rheumatoid factor; ESR, erythrocyte sedimentation rates; ANA, antinuclear antibody; DAS28, disease activity score 28; HAQ, Health assessment questionnaire. 
$100 \mu \mathrm{L}$ biotin-antibody was added before incubating for $1 \mathrm{~h}$ at $37^{\circ} \mathrm{C}$. Third, liquid was removed and the well was washed, followed with the addition of $100 \mu \mathrm{L}$ horseradish Peroxidase-avidin for $1 \mathrm{~h}$ at $37^{\circ} \mathrm{C}$. Fourth, removing the liquid, adding $90 \mu \mathrm{L} \mathrm{3,3}, 5,5^{\prime}$-Tetramethylbenzidine to the well was performed, which was then incubated at $37^{\circ} \mathrm{C}$ for 0.5 h. Finally, $50 \mu \mathrm{L}$ stop solution was added, and the plate was read at $450 \mathrm{~nm}$ by a microplate reader. The minimum detecting level of GDF-15 was $1.95 \mathrm{pg} / \mathrm{mL}$. Intra-assay and inter-assay reliability of GDF-15 assay were 5.5\% and $6.5 \%$, respectively. Individual samples were examined in duplicate.

\section{Single Nucleotide Polymorphism Genotyping}

DNA was extracted from PBMC isolated from each participant according to manufacturer's instruction (TIANGEN, Beijing, China). The concentration and purity of each DNA sample was examined, and qualified DNA was used for genotyping. We selected four single nucleotide polymorphisms (SNPs) in the GDF-15 gene with several conditions, including pairwise tagging of HapMap population with $r^{2} \geq 0.8$, a minor allele frequency $\geq 5 \%$, Chinese Han Beijing ethnicity. At last, polymorphisms including rs1055150, rs1058587, rs3787023 and rs4808793 were selected. The KASP method was conducted for genotyping (Gene Company, Shanghai, China), ${ }^{12}$ and the primer information for the four polymorphisms is listed in Supplementary Table 1.

\section{Data Statistics}

All the data were analyzed by SPSS software. Quantitative data were described as median (interquartile range) if the data were not normally distributed. Qualitative data were described as frequency or proportion. Two groups of data were compared by Wilcoxon rank-sum test, and correlation analysis was conducted by Spearman correlation test. Logistic regression was used for discussing the relationship of polymorphisms and RA risk, including odds ratio (OR) and 95\% confidence interval (CI). The receiver operating characteristic (ROC) curve discussed the potential of plasma GDF-15 as the marker for RA. A $P$-value less than 0.05 was significant.

\section{Results}

\section{Increased Plasma Levels of GDF-I5 in RA Patients}

There were elevated plasma levels of GDF-15 in RA patients as compared to that in healthy controls $(P<0.001$, Figure 1). We also analyzed plasma levels of GDF-15 according to clinical and laboratory characteristics. Results showed that RA patients with positive CRP had higher plasma levels of GDF-15 as compared to that in patients with negative CRP $(P<0.001)$. However, RA patients with early disease did not show a significant difference of plasma levels of GDF-15 as compared to that in patients with long disease $(P=0.829)$. Similarly, RA patients with positive ANA had comparable plasma levels of GDF-15 as compared to that in patients with negative ANA $(P=0.057)$. Correlation analysis revealed that plasma levels of GDF-15 were significantly related to DAS28 (ESR) $\left(\mathrm{r}_{\mathrm{s}}=0.426, P<0.001\right)$ and were not related to RF levels $\left(\mathrm{r}_{\mathrm{s}}=0.020, P=0.875\right)$. ROC analysis showed that the AUC was 0.692 when plasma levels of GDF-15 in RA patients were compared to that in controls (Figure 1).

\section{Relationship between GDF-I5 Gene Polymorphisms and RA Risk}

This study evaluated GDF-15 gene polymorphisms (rs1055150, rs1058587, rs3787023, and rs4808793) and RA risk. Results found that frequencies of GG, GC, GG+GC genotypes, and $\mathrm{G}$ allele of rs 1055150 were significantly higher in RA patients as compared to those in healthy controls ( $\mathrm{GG}$ versus $\mathrm{CC}, \mathrm{OR}=1.619,95 \% \mathrm{CI}=1.020-2.568, P=0.041$; $\mathrm{GC}$ versus $\mathrm{CC}, \mathrm{OR}=1.458,95 \% \mathrm{CI}=1.057-2.010, P=0.022 ; \mathrm{GG}+\mathrm{GC}$ versus $\mathrm{CC}, \mathrm{OR}=1.495,95 \% \mathrm{CI}=1.104-2.022, P=0.009$; $\mathrm{G}$ versus $\mathrm{C}, \mathrm{OR}=1.330,95 \% \mathrm{CI}=1.069-1.654, P=0.011$, Table 2). Similarly, frequencies of CC genotype, $\mathrm{C}$ allele in rs3787023 were significantly higher in RA patients as compared to those in controls (CC versus TT, OR=1.838, 95\% $\mathrm{CI}=1.022-3.305, P=0.042$; $\mathrm{C}$ versus $\mathrm{T}, \mathrm{OR}=1.310,95 \% \mathrm{CI}=1.040-1.651, P=0.022$, Table 2). Gene polymorphisms (rs1058587 and rs4808793) were not related to RA risk, neither discussing frequencies of the genotypes nor alleles (Table 2). 

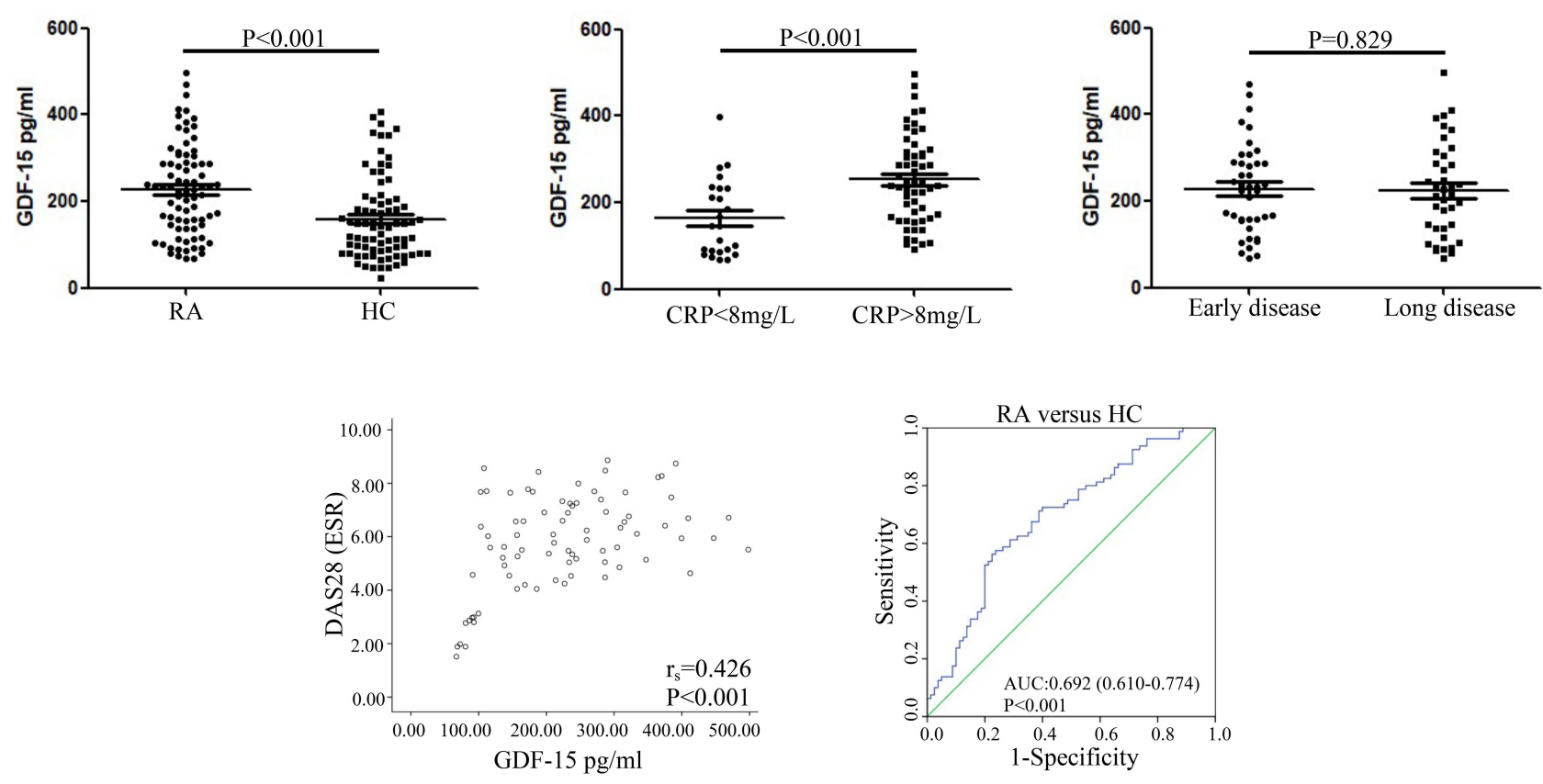

Figure I Plasma levels of GDF-I5 in RA patients. Plasma levels of GDF-I5 were tested by enzyme linked immunosorbent assay in 80 RA patients and 80 healthy controls. Differences of plasma levels of GDF-I5 were compared between patients and controls. The association of plasma levels of GDF-I5 in RA patients with positive, negative CRP, or with early disease, long disease was discussed. Correlation analysis of plasma levels of GDF-15 with disease activity score was conducted by Spearman's test. A receiver operating characteristic curve evaluated the potential of plasma GDF-I5 as the disease marker. Comparison between two groups of data was analyzed by Wilcoxon rank-sum test.

To reveal the association of GDF-15 gene polymorphisms with RA susceptibility, we further discussed the relationship between GDF-15 gene polymorphisms with RA clinical, laboratory characteristics. Results showed that the number of tender joints was comparable between RA patients carrying $\mathrm{GG}+\mathrm{GC}$ genotype as compared to that in RA patients carrying CC genotype for rs1055150 ( $P=0.503$, Supplementary Table 2). Similarly, the other characteristics, including number of swollen joints, levels of ESR, CRP, RF, and HAQ score, were not significantly related to rs 1055150. Furthermore, polymorphisms including rs1058587, rs3787023, and rs4808793 were not significantly related to RA clinical, laboratory characteristics (Supplementary Table 2).

\section{Effect of GDF-I5 Gene Polymorphisms on Plasma Levels of GDF-I5}

We evaluated the potential effect of GDF-15 gene polymorphisms on plasma levels of GDF-15. According to polymorphism rs 1055150, RA patients carrying the GG genotype, GC genotype, and CC genotype had comparable plasma levels of GDF-15 ( $P=0.519$, Figure 2). Similarly, for rs1058587, RA patients carrying the GG genotype, GC genotype, and CC genotype had comparable plasma levels of GDF-15 as well. Moreover, for rs3787023 and rs4808793, there was no significant difference among the genotypes regarding plasma levels of GDF-15 ( $P=0.561, P=0.909$, Figure 2$)$.

\section{Discussion}

This study aims to evaluate plasma levels of GDF-15 in RA patients and discuss the relationship of GDF-15 gene polymorphisms with RA susceptibility in a Chinese Han population. A case-control study with 455 RA patients and 455 healthy controls was conducted, along with sex- and age-matching. We found that RA patients had much higher plasma levels of GDF-15 as compared to that in healthy controls. Patient with positive CRP also showed higher expression of plasma levels of GDF-15 when compared with that in patients with negative CRP. Interestingly, plasma levels of GDF-15 was positively related to DAS28 (ESR), and the AUC was 0.692 . These findings suggested that GDF-15 may correlate with RA pathogenesis. Our findings are similar to several studies discussing serum levels of GDF-15 in RA patients. ${ }^{10-12}$ A study evaluated serum GDF-15 in Iran RA patients, showing that serum GDF-15 may be a biomarker to predict high disease activity. ${ }^{13}$ Another study discussed serum GDF-15 in Turkish RA patients, reporting that serum GDF-15 was 
Table 2 Frequencies of Alleles and Genotypes for Four Single-Nucleotide Polymorphisms in GDF-I5 Gene in Rheumatoid Arthritis Patients and Healthy Controls

\begin{tabular}{|c|c|c|c|c|}
\hline Polymorphism & $\begin{array}{l}\text { Rheumatoid Arthritis } \\
\text { (n, \%) }\end{array}$ & $\begin{array}{l}\text { Healthy Controls } \\
(\mathrm{n}, \%)\end{array}$ & OR $(95 \% \mathrm{CI})$ & $P$-value \\
\hline rsI055I50 & & & & \\
\hline GG & $56(|2.3|)$ & $72(15.82)$ & $1.619(1.020-2.568)$ & 0.041 \\
\hline GC & $189(4 \mid .54)$ & $217(47.69)$ & $1.458(1.057-2.010)$ & 0.022 \\
\hline$G G+G C$ & $245(53.85)$ & $289(63.5 \mathrm{I})$ & $1.495(1.104-2.022)$ & 0.009 \\
\hline $\mathrm{CC}$ & $210(46.15)$ & $166(36.49)$ & - & \\
\hline G & $300(32.97)$ & $360(39.56)$ & $1.330(1.069-1.654)$ & 0.011 \\
\hline $\begin{array}{l}\text { C } \\
\text { rs1058587 }\end{array}$ & $610(67.03)$ & $550(60.44)$ & - & \\
\hline GG & $23 \mid(50.77)$ & 221 (48.57) & $0.605(0.328-I .115)$ & 0.107 \\
\hline GC & $199(43.74)$ & $195(42.86)$ & $0.621(0.335-I .15 I)$ & 0.130 \\
\hline$G G+G C$ & $430(94.5 I)$ & $416(91.43)$ & $0.612(0.338-1.110)$ & 0.106 \\
\hline $\mathrm{CC}$ & 25 (5.49) & $39(8.57)$ & - & \\
\hline G & $662(72.75)$ & $637(70.00)$ & $0.876(0.694-1.104)$ & 0.261 \\
\hline $\begin{array}{l}\text { C } \\
\text { rs3787023 }\end{array}$ & $248(27.25)$ & $273(30.00)$ & - & \\
\hline $\mathrm{CC}$ & 207 (45.49) & $242(53.19)$ & $1.838(1.022-3.305)$ & 0.042 \\
\hline CT & $205(45.05)$ & I $86(40.88)$ & $\mathrm{I} .422(0.787-2.57 \mathrm{I})$ & 0.244 \\
\hline $\mathrm{CC}+\mathrm{CT}$ & $412(90.55)$ & $428(94.07)$ & 1.631 (0.924-2.879) & 0.092 \\
\hline TT & $43(9.45)$ & $27(5.93)$ & - & \\
\hline C & $619(68.02)$ & $670(73.63)$ & $1.310(1.040-1.651)$ & 0.022 \\
\hline $\begin{array}{l}\mathrm{T} \\
\text { rs4808793 }\end{array}$ & 291 (31.98) & $240(26.37)$ & - & \\
\hline GG & $42(9.23)$ & $49(10.77)$ & I.I24 (0.669-I.886) & 0.660 \\
\hline GC & $208(45.7 I)$ & $189(41.54)$ & $0.857(0.627-I .172)$ & 0.235 \\
\hline$G G+G C$ & $250(54.95)$ & $238(52.3 \mathrm{I})$ & $0.902(0.670-1.214)$ & 0.495 \\
\hline $\mathrm{CC}$ & $205(45.05)$ & $217(47.69)$ & - & \\
\hline G & $291(31.98)$ & $287(31.54)$ & $0.980(0.783-1.228)$ & 0.863 \\
\hline C & $619(68.02)$ & $623(68.46)$ & - & \\
\hline
\end{tabular}

Abbreviations: OR, odd ratio; $95 \% \mathrm{Cl}, 95 \%$ confidence interval.

elevated in RA patients when compared to that in controls. ${ }^{11}$ RA patients with active disease activity had higher serum levels of GDF-15 compared to that in patients with less active disease activity, and serum levels of GDF-15 were positively related to ESR levels and DAS28 score. ${ }^{14}$ All the findings indicated that GDF-15 may relate to RA pathogenesis, such as damage of different joints. Since these findings showed a significant association of GDF-15 and RA, what is the mechanism that GDF-15 contributes to RA development? This needs to be discussed in the future with functional studies, for instance, usage of arthritis mice models to discuss effects of GDF-15 in onset or development of the disease. In a study which discussed the association of GDF-15 with rheumatic diseases using published genetic data with a Mendelian randomization (MR) design, Ye et al ${ }^{6}$ found that there was no evidence of a causal association between circulating GDF-15 levels and risk of RA. Discrepancy between the positive findings from our study, previous studies and the largely negative data from Ye et al may correlate with several reasons. First, Ye et al used published data for further study with Mendelian randomization (MR) design, while the Mendelian randomization (MR) design is a method to discuss the association of circulating levels of GDF-15 with RA in the study. Indeed, the other studies examined levels of GDF-15 in RA patients by laboratory test. The original results may be more reliable. Second, if the author wants to discuss an association of GDF-15 levels with RA risk, a meta-analysis may be conducted, which may be more persuasive than a single-center study such as our study. However, all the published data show that circulating levels of GDF-15 were increased in RA patients, as discussed above; therefore, a meta-analysis is not necessary. Nevertheless, the direct method 

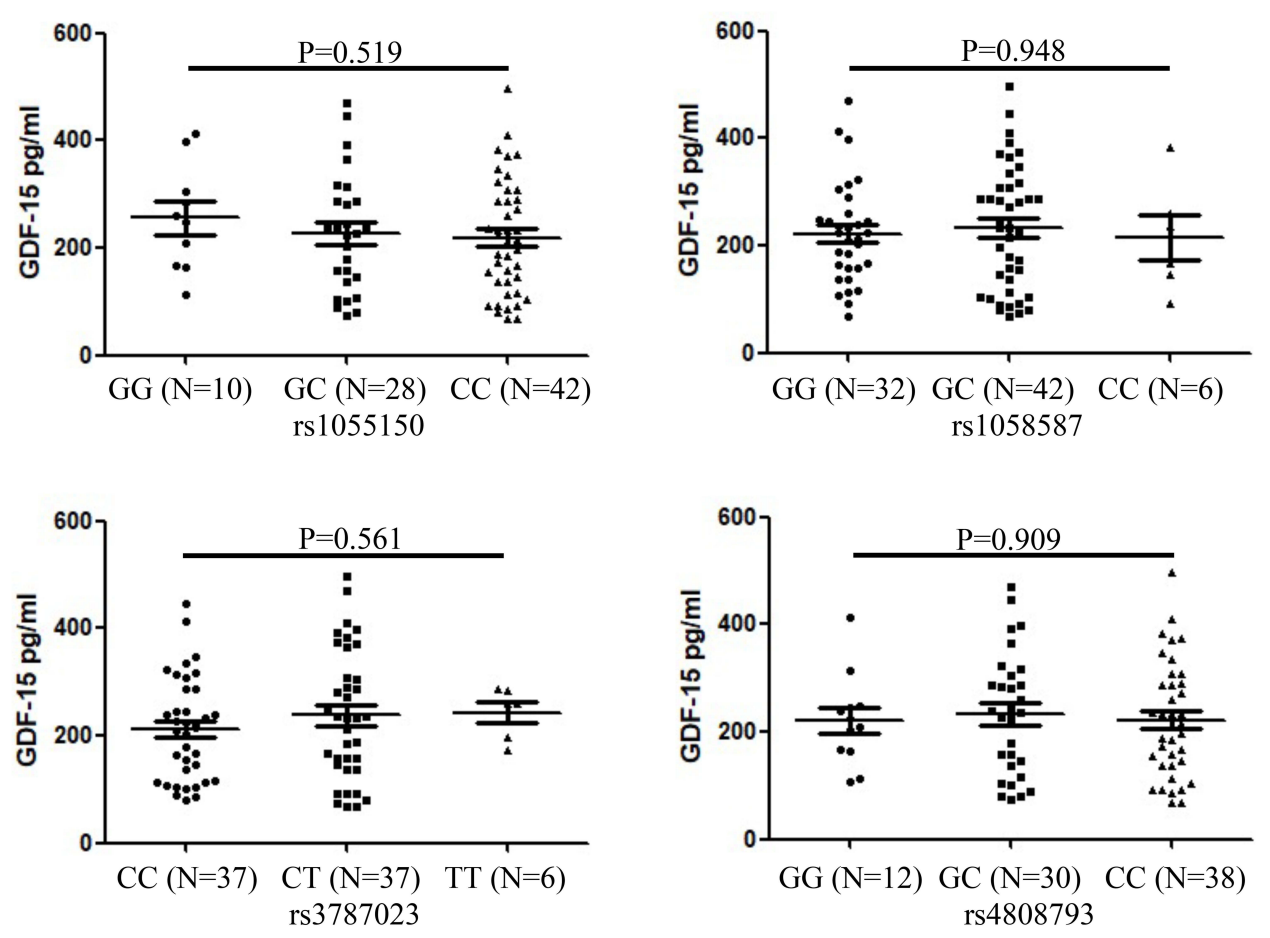

Figure 2 Association of GDF-15 gene polymorphisms with plasma levels of GDF-15. Eighty RA patients examined plasma levels of GDF-15, and the patients tested GDF-15 gene polymorphisms ( $r$ 1055150, rs 1058587, rs3787023 and rs4808793) as well. The potential effect of polymorphisms on plasma levels of GDF-15 was evaluated by comparing plasma levels of GDF-15 among three different genotypes, including GG, GC, and CC genotypes for rs 1055 I50, rs 1058587, and rs4808793 and CC, CT, and TT genotypes for rs3787023. Three groups of data were discussed according to Kruskal-Wallis test.

to discuss whether GDF-15 contributes RA pathogenesis is to use an arthritis model by which the GDF-15 gene is deficient or overexpressed in the future.

There are several studies having evaluated the relationship of GDF-15 gene polymorphisms with human diseases. ${ }^{12-15}$ Xiang et $\mathrm{al}^{15}$ recruited 298 stroke patients and 303 healthy controls ofChinese origin to evaluate the association of polymorphisms rs3787023, rs1055150, and rs1804826 with stroke risk by snapshot SNP genotyping assays and sequencing. They found that TT, GT, and TT+GT genotypes in rs1804826 were significantly related to stroke risk, and the rs $1804826 \mathrm{~T}$ allele was significantly related to elevated risk of stroke as well. By contrast, neither genotypes nor alleles of rs3787023 or rs 1055150 were correlated with stroke risk. ${ }^{15}$ Another study conducted on 448 Chinese coronary artery disease patients found that frequencies of GG, GC, and CC genotype in rs4808793 were not different between patients and controls using a ligase detection reaction method. ${ }^{16}$ For patients with colorectal cancer, the frequency of GG+GC genotype in rs 1058587 polymorphism was elevated in 349 colorectal cancer patients of Chinese origin when compared to that in controls by pyro-sequencing method to genotype rs $1058587 .{ }^{17}$ Colorectal cancer patients with GG+GC genotype in rs 1058587 showed elevated risk of death from colon cancer than the patients carrying homozygote CC genotype. ${ }^{17}$ The study discussed prostate cancer in 819 Australia patients with sequencing method to genotype rs $1058587,{ }^{18}$ showing that neither genotypes nor allele were related to prostate cancer risk. However, there was an elevated risk of death from prostate cancer in patients carrying the $\mathrm{G}$ allele than patients carrying the $\mathrm{C}$ allele. ${ }^{18}$ When discussing the association of GDF-15 gene polymorphisms with RA risk, a study recruited 723 Swedish RA patients with sequencing method to genotype rs $1058587 .{ }^{19}$ The authors reported that the distribution of genotypes GG and GC of rs1058587 differed significantly between RA patients and controls. ${ }^{19}$ Moreover, Amaya-Amaya et $\mathrm{al}^{20}$ evaluated $\mathrm{rs} 1058587$ polymorphism with RA risk in 310 Latin Americans by TaqMan allelic discrimination assay. They found that significant differences concerning rs 1058587 polymorphism were not obtained between RA patients and controls. ${ }^{20}$ Our findings were partly similar to the above findings. For example, regarding rs4808793, our results also showed no significant differences between RA patients and controls. With respect to rs3787302, we found significant results when comparing frequencies of CC genotype, $\mathrm{C}$ allele between RA patients and controls. For rs 1058587, our findingss were similar to the findings in 
Latin American RA patients, but not the same as findings in Swedish RA patients. Considering the reasons, the differences may correlate with several clues. First, different diseases have different pathogenesis. Therefore, when discussing the same polymorphism, the results may be different, such as findings for rs3787023 in stroke, RA. Second, development of different diseases may correlate with different ethnicity. For instance, the study discussed rs1058587 in Latin American RA patients, showing with no significant results, whereas the study assessed rs 1058587 in Swedish RA patients, revealing with significant results. Third, different sample sizes and methods to genotype the polymorphisms may have different findings as discussed above. Thus, to better understand correlation between GDF-15 gene polymorphisms and RA susceptibility, further studies with larger sample sizes in different ethnicity and the same method should be conducted. Fourth, different polymorphisms may correlate with different disease, for example, rs1804826 was related to stroke, where we did not analyze this polymorphism. On the other hand, we found rs1055150, rs3787023 polymorphisms were related to RA risk, but these polymorphisms were not related to stroke.

It is important to discuss the effect of polymorphism on mRNA expression or protein expression for a gene since aberrant function of a gene or protein sometimes is regulated by mutated base, which may affect transcription to mRNA and translation of protein. ${ }^{21}$ We discussed the association of the four polymorphisms and plasma levels of GDF-15. There was no significant result neither discussing plasma levels of GDF-15 in GG, GC, or CC genotype for rs1055150, nor for the other three polymorphisms. However, this study only discussed the relationship of GDF-15 levels and the four polymorphisms. Whether there are some other polymorphisms that may affect GDF-15 expression needs to be discussed in the future. Furthermore, a functional study of the four polymorphisms will need to confirm whether these polymorphisms can affect expression of GDF-15 in RA patients.

It is interesting to address the possible mechanism of action of GDF-15 in pathogenesis of RA. A similar statement about the genetic changes in the gene for GDF-15 is useful to show how may it affect RA. A C to G substitution leading to histidine to aspartic acid change at codon 202 of the precursor GDF-15 protein is called H6D (rs1058587), because the amino change is at position 6 of the mature GDF-15 protein. ${ }^{22}$ A study with prostate cancer patients showed that rs $1058587 \mathrm{G}$ allele was related to reduced risk of developing prostate cancer. ${ }^{8}$ Human prostate cancer DU145 cells expressing the rs $1058587 \mathrm{G}$ allele or $\mathrm{C}$ allele were injected into nude mice, showing that prostate cancer DU145 cells expressing the rs $1058587 \mathrm{G}$ allele suppressed prostate tumorigenesis by suppressing IGF-1 and cyclin D1 expression. ${ }^{23}$ The transcription activity of rs 4808793 G-containing construct was increased 1.45-fold at baseline and 1.73 -fold after stimulation with phenylephrine when compared to the rs4808793 C construct, suggesting that rs4808793 G allele associated with an increase in GDF-15 levels in hypertensive patients. ${ }^{24} \mathrm{~A}$ meta-analysis showed that hypertension increased cardiovascular risk in patients with RA. ${ }^{25}$ Since the rs $4808793 \mathrm{G}$ allele was related to high expression of GDF15 in hypertensive patients, it is hypothesized that the rs $4808793 \mathrm{G}$ allele may contribute to elevated expression of GDF15 in RA patients, especially RA patients complicated with hypertension. GDF-15 is required for Th2 cytokines (IL-4, IL-13) induced glucose intolerance in mice. ${ }^{26}$ It is known that the Th2 cell and related cytokines are important for RA pathogenesis and development. ${ }^{27}$ Thus, GDF-15 may regulate Th2 cells and then affect RA pathogenesis. Moreover, there was increased expression of CXCR3 in activated T-cells from GDF-15 deficient mice. ${ }^{28}$ The CXCR3 antagonist has anti-arthritic effects on collagen-induced arthritis by inhibiting B-cell mediated inflammation, reducing the percentage of Th1, Th17 and Th22 cells and increasing the proportion of Treg cells. ${ }^{29,30}$ Collectively, GDF-15 may involve RA, which needs to be confirmed in the future.

Some limitations in this study need to be realized. First, clinical and laboratory characteristics of RA were not enough. Second, RA is a complex disease, where gene-gene interaction and gene-environment interaction may correlate with pathogenesis of the disease. These discussions may be considered in the future. Third, larger sample sizes and more polymorphisms may be conducted to confirm the current findings.

In conclusion, the present study showed that plasma levels of GDF-15 were elevated in RA patients and GDF-15 gene polymorphisms were related to RA risk in the Chinese Han population.

\section{Abbreviations}

RA, rheumatoid arthritis; GDF-15, growth and differentiation factor 15 ; TGF- $\beta$, transforming growth factor- $\beta$; ACR/ EULAR, American College of Rheumatology and European League Against Rheumatism; DAS28, disease activity score 
28; ESR, erythrocyte sedimentation rate; CRP, C-reactive protein; RF, rheumatoid factor; ANA, antinuclear antibody; HAQ, health assessment questionnaire.

\section{Data Sharing Statement}

Datasets are available from the corresponding author on reasonable request.

\section{Author Contributions}

All authors made a significant contribution to the work reported, whether that is in the conception, study design, execution, acquisition of data, analysis and interpretation, or in all these areas; took part in drafting, revising, or critically reviewing the article; gave final approval of the version to be published; have agreed on the journal to which the article has been submitted; and agree to be accountable for all aspects of the work.

\section{Funding}

The study was supported by Program of Sichuan Provincial Science and Technology-Luzhou Municipal People's Government-Luzhou Medical College (201410074).

\section{Disclosure}

The authors declare that they have no conflicts of interest.

\section{References}

1. Humby F, Durez P, Buch MH, et al. Rituximab versus tocilizumab in anti-TNF inadequate responder patients with rheumatoid arthritis (R4RA): 16-week outcomes of a stratified, biopsy-driven, multicentre, open-label, phase 4 randomised controlled trial. Lancet. 2021;397(10271):305-317. doi:10.1016/S0140-6736(20)32341-2

2. Dedmon LE. The genetics of rheumatoid arthritis. Rheumatology. 2020;59(10):2661-2670. doi:10.1093/rheumatology/keaa232

3. Lodi RS, Yu B, Xia L, Liu F. Roles and Regulation of Growth differentiation factor-15 in the Immune and tumor microenvironment. Hum Immunol. 2021;82:937-944. doi:10.1016/j.humimm.2021.06.007

4. Wang D, Day EA, Townsend LK, Djordjevic D, Jørgensen SB, Steinberg GR. GDF15: emerging biology and therapeutic applications for obesity and cardiometabolic disease. Nat Rev Endocrinol. 2021;17(10):592-607. doi:10.1038/s41574-021-00529-7

5. Wischhusen J, Melero I, Fridman WH. Growth/differentiation factor-15 (GDF-15): from biomarker to novel targetable immune checkpoint. Front Immunol. 2020;11:951. doi:10.3389/fimmu.2020.00951

6. Ye D, Liu B, He Z, et al. Assessing the associations of growth differentiation factor 15 with rheumatic diseases using genetic data. Clin Epidemiol. 2021;13:245-252. doi:10.2147/CLEP.S305024

7. Brown DA, Ward RL, Buckhaults P, et al. MIC-1 serum level and genotype: associations with progress and prognosis of colorectal carcinoma. Clin Cancer Res. 2003;9(7):2642-2650.

8. Lindmark F, Zheng SL, Wiklund F, et al. H6D polymorphism in macrophage-inhibitory cytokine-1 gene associated with prostate cancer. $J$ Natl Cancer Inst. 2004;96(16):1248-1254. doi:10.1093/jnci/djh227

9. Neogi T, Aletaha D, Silman AJ, et al. The 2010 American college of Rheumatology/European league against rheumatism classification criteria for rheumatoid arthritis: Phase 2 methodological report. Arthritis Rheum. 2010;62(9):2582-2591. doi:10.1002/art.27580

10. Prevoo ML, van 't Hof MA, Kuper HH, Van leeuwen MA, van de Putte LB, van Riel PL. Modified disease activity scores that include twenty-eight-joint counts. Development and validation in a prospective longitudinal study of patients with rheumatoid arthritis. Arthritis Rheum. 1995;38(1):44-48. doi:10.1002/art.1780380107

11. Xu WD, Su LC, He CS, Huang AF. Plasma interleukin-38 in patients with rheumatoid arthritis. Int Immunopharmacol. 2018;65:1-7. doi:10.1016/j. intimp.2018.09.028

12. Xu WD, Wu Q, He YW, et al. Gene polymorphisms of LGALS2, LGALS3 and LGALS9 in patients with rheumatoid arthritis. Cell Immunol. 2021;368:104419. doi:10.1016/j.cellimm.2021.104419

13. Esalatmanesh K, Fayyazi H, Esalatmanesh R, Khabbazi A. The association between serum levels of growth differentiation factor-15 and rheumatoid arthritis activity. Int J Clin Pract. 2020;74(9):e13564. doi:10.1111/ijcp.13564

14. Tanrıkulu O, Sarıyldız MA, Batmaz İ, et al. Serum GDF-15 level in rheumatoid arthritis: relationship with disease activity and subclinical atherosclerosis. Acta Reumatol Port. 2017;42(1):66-72.

15. Xiang Y, Zhang T, Guo J, Peng YF, Wei YS. The association of growth differentiation factor-15 gene polymorphisms with growth differentiation factor-15 serum levels and risk of ischemic stroke. J Stroke Cerebrovasc Dis. 2017;26(10):2111-2119. doi:10.1016/j. jstrokecerebrovasdis.2017.04.031

16. Chen Z, Xie F, Ma G, Feng Y, Qian Q, Liu N. Study of the association between growth differentiation factor 15 gene polymorphism and coronary artery disease in a Chinese population. Mol Biol Rep. 2011;38(8):5085-5091. doi:10.1007/s11033-010-0655-4

17. Wang J, Li W, Wang Y, et al. The H6D genetic variation of GDF15 is associated with genesis, progress and prognosis in colorectal cancer. Pathol Res Pract. 2015;211(11):845-850. doi:10.1016/j.prp.2015.08.004

18. Hayes VM, Severi G, Southey MC, et al. Macrophage inhibitory cytokine-1 H6D polymorphism, prostate cancer risk, and survival. Cancer Epidemiol Biomarkers Prev. 2006;15(6):1223-1225. doi:10.1158/1055-9965.EPI-06-0063 
19. Ärlestig L, Rantapää-Dahlqvist S. Polymorphisms of the genes encoding CD40 and growth differentiation factor 15 and in the 9p21.3 region in patients with rheumatoid arthritis and cardiovascular disease. J Rheumatol. 2012;39(5):939-945. doi:10.3899/jrheum.111336

20. Amaya-Amaya J, Rojas-Villarraga A, Molano-Gonzalez N, Montoya-Sánchez L, Nath SK, Anaya JM. GDF15(MIC1) H6D polymorphism does not influence cardiovascular disease in a latin American population with rheumatoid arthritis. J Immunol Res. 2015;2015:270763. doi:10.1155/2015/ 270763

21. Xu WD, Liu XY, Su LC, Huang AF. Association of MASP2 levels and MASP2 gene polymorphisms with systemic lupus erythematosus. $J$ Cell Mol Med. 2020;24(18):10432-10443. doi:10.1111/jcmm.15656

22. Fairlie WD, Russell PK, Wu WM, et al. Epitope mapping of the transforming growth factor-beta superfamily protein, macrophage inhibitory cytokine-1 (MIC-1): identification of at least five distinct epitope specificities. Biochemistry. 2001;40(1):65-73. doi:10.1021/bi001064p

23. Wang X, Chrysovergis K, Bienstock RJ, Shim M, Eling TE. The H6D variant of NAG-1/GDF15 inhibits prostate xenograft growth in vivo. Prostate. 2012;72(6):677-689. doi:10.1002/pros.21471

24. Wang X, Yang X, Sun K, et al. The haplotype of the growth-differentiation factor 15 gene is associated with left ventricular hypertrophy in human essential hypertension. Clin Sci. 2009;118(2):137-145. doi:10.1042/CS20080637

25. Baghdadi LR, Woodman RJ, Shanahan EM, Mangoni AA. The impact of traditional cardiovascular risk factors on cardiovascular outcomes in patients with rheumatoid arthritis: a systematic review and meta-analysis. PLoS One. 2015;10(2):e0117952. doi:10.1371/journal.pone.0117952

26. Lee SE, Kang SG, Choi MJ, et al. Growth differentiation factor 15 mediates systemic glucose regulatory action of t-helper type 2 cytokines. Diabetes. 2017;66(11):2774-2788. doi:10.2337/db17-0333

27. Bridgewood C, Newton D, Bragazzi N, Wittmann M, McGonagle D. Unexpected connections of the IL-23/IL-17 and IL-4/IL-13 cytokine axes in inflammatory arthritis and enthesitis. Semin Immunol. 2021;101520. doi:10.1016/j.smim.2021.101520

28. Moschovaki-Filippidou F, Steiger S, Lorenz G, et al. Growth differentiation factor 15 ameliorates anti-glomerular basement membrane glomerulonephritis in mice. Int J Mol Sci. 2020;21(19):6978. doi:10.3390/ijms21196978

29. Bakheet SA, Alrwashied BS, Ansari MA, et al. CXC chemokine receptor 3 antagonist AMG487 shows potent anti-arthritic effects on collagen-induced arthritis by modifying B cell inflammatory profile. Immunol Lett. 2020;225:74-81. doi:10.1016/j.imlet.2020.06.014

30. Bakheet SA, Ansari MA, Nadeem A, et al. CXCR3 antagonist AMG487 suppresses rheumatoid arthritis pathogenesis and progression by shifting the Th17/Treg cell balance. Cell Signal. 2019;64:109395. doi:10.1016/j.cellsig.2019.109395

Journal of Inflammation Research

Dovepress

\section{Publish your work in this journal}

The Journal of Inflammation Research is an international, peer-reviewed open-access journal that welcomes laboratory and clinical findings on the molecular basis, cell biology and pharmacology of inflammation including original research, reviews, symposium reports, hypothesis formation and commentaries on: acute/chronic inflammation; mediators of inflammation; cellular processes; molecular mechanisms; pharmacology and novel anti-inflammatory drugs; clinical conditions involving inflammation. The manuscript management system is completely online and includes a very quick and fair peer-review system. Visit http://www.dovepress.com/testimonials.php to read real quotes from published authors.

Submit your manuscript here: https://www.dovepress.com/journal-of-inflammation-research-journal 